\title{
FATORES DE SUPORTE AO AUMENTO DA PRODUTIVIDADE DA TECNOLOGIA DA INFORMAÇÃO
}

\section{FACTORS OF SUPPORT TO THE INCREASE OF PRODUCTIVITY OF INFORMATION TECHNOLOGY}

\author{
Sergio Luiz dos Santos ${ }^{1}$; Fernando José Barbin Laurindo \\ ${ }^{1}$ PRO-POLI-USP, sergio.santos@poli.usp.br \\ ${ }^{2}$ PRO-POLI-USP, fjblau@usp.br
}

\author{
Recebido para publicação em: 04/04/2005 \\ Aceito para publicação em: 21/08/2005
}

\section{Resumo}

O impacto da Tecnologia da Informação (TI) na produtividade do nível gerencial das organizações é uma questão de grande importância que vem sendo extensivamente pesquisada desde a disseminação da TI nas empresas na década de 70. Esse esforço se justifica pela preocupação das empresas em relação às pressões simultâneas sofridas, tais como a globalização, mudanças mundiais no mercado de mão de obra, sofisticação da demanda dos consumidores, esgotamento de mercados inexplorados, entre outros, vide Caldas (1999). No entanto, medir a produtividade do fator TI não é uma tarefa simples, pois está sujeita a variações subjetivas de cada administrador e varia para cada empresa. As soluções encontradas costumam ser generalizadas para vários casos em segmentos totalmente diferentes, o que gera distorções e resultados insatisfatórios. $O$ objeto de estudo deste artigo é o conjunto de estruturas internas à organização capazes de extrair benefícios da TI em favor da produtividade. A pesquisa envolve um levantamento teórico e um estudo de caso em uma empresa de produção e prestação de serviços na área de cromatografia a gás na cidade de São Paulo. Como resultado, chegou-se a uma lista de verificação dos elementos necessários para impulsionar a produtividade através do auxilio da TI.

Palavras-chave: Tecnologia da Informação, Produtividade, Processos de Negócio

\section{Introdução}

O presente trabalho apresenta um estudo de caso realizado para levantar como considerar os fatores relevantes dentro das organizações que devem ser estruturados para aumento da produtividade viabilizada pelo uso da TI.

Inicialmente será introduzido um conceito de produtividade e como tem sido seu desempenho no conjunto das empresas. Neste ponto, será feito um paralelo entre o aumento de 
produtividade no Brasil e certos fatores que também impulsionam tal produtividade, com o intuito de encontrar outros fatores que alinhados à TI contribuíram para o incremento da produtividade nos últimos anos.

A seguir serão enumerados fatores que apóiam a TI e que, se bem implementados, são pilares para a atuação da TI como elemento de melhoria da produtividade dentro da empresa.

A importância desta análise é que não só um, mas um conjunto de fatores é necessário, tais como processos bem planejados e profissionais capacitados, de forma que a sinergia de todos juntos promove o sucesso da implantação da TI. Tenta-se mostrar que os investimentos em TI não devem ser realizados sem uma séria composição, dentro da organização, de um quadro importante para o retorno dos investimentos em TI.

Assim, não há uma maneira de "garantir" que o investimento em TI possa sempre retornar benefícios devido à especificidade de cada empresa, mas alguns pontos devem ser analisados nessa situação, como: utilização de processos de negócios adequados; análise da intensidade de informação e inovação tecnológica da empresa; erros de medição (uso de indicadores inadequados); especificidades de áreas dentro das empresas e das próprias empresas.

\section{Produtividade e tecnologia da informação}

Produtividade possui diferentes significados para diferentes pessoas, sob a luz de suas experiências próprias e interesses conforme Machado (1964). Tradicionalmente é o quociente de uma produção por um dos fatores de produção, tais como tempo, matéria prima e recursos financeiros. Atualmente, este conceito tradicional de produtividade não se ajusta ao mercado competitivo, no qual o sistema de produção em massa e de baixa flexibilidade dá lugar a processos mais intensivos em informação conforme Porter e Millar (1985) e organizações altamente sensíveis ao mercado mundial, vide Weill et al (2002).

Nesse sentido, um conceito mais amplo de produtividade inclui eficiência e eficácia, no qual eficiência é a medida do desempenho do processo de conversão das entradas em saídas, enquanto eficácia é a medida do grau em que as saídas satisfazem os requisitos, conforme apresentado por Carvalho e Laurindo (2003).

Para Laurindo (2002), dentre as tecnologias adotadas para o aumento de produtividade nos últimos anos, a TI é vista como fator de viabilização da competição em abrangência mundial, bem como de criação de novas estratégias de negócios, de novas estruturas organizacionais e de novas formas de relacionamento entre empresas e entre empresas e seus consumidores. 
Porter e Millar (1985) afirmam que se aumenta a produtividade desempenhando as atividades de valor (atividades ordenadas dentro da organização que agregam valor ao produto final para o cliente) a um custo menor ou com orientação à diferenciação, e a TI é ferramenta essencial para esta mudança. Para Carr (2003) a TI é infra-estrutura essencial para o desenvolvimento intra e inter organizacional. Para Farrel (2004), o conceito de produtividade envolve o conceito de inovação, buscando novos bens e serviços de maior valor adicionado, e de agregação de valor nos bens atuais.

\section{A produtividade no Brasil}

Muitos textos referem-se à aplicação da TI e ao aumento da eficiência nas empresas norteamericanas, mas a relação causa-efeito na maioria das vezes não é a mesma no Brasil, e a produtividade pode não ser explicada de forma adequada.

Muitas empresas norte americanas consideraram a reengenharia o trunfo produtivo dos anos 90. No Brasil, é importante ressaltar que os aumentos na produtividade industrial foram obtidos dentre outras formas, através de investimentos em técnicas gerenciais e organizacionais. Para Feijó e Carvalho (1994), basicamente, tais técnicas seguiram a tendência mundial de enxugamento do quadro de empregados, da elevação do nível de qualificação da mão-de-obra e inserção tecnológica. Para estes autores, os fatores para o aumento de produtividade foram: ajuste recessivo (abertura de mercado e mudança de governo); globalização; reestruturação produtiva, com técnicas como programas de qualidade, JIT e reengenharia, com paradigma tecnológico gerencial altamente poupador de mão de obra. Portanto, o aumento individual da produtividade dos funcionários explica somente parte dos resultados obtidos neste período, e originou-se do aumento da qualificação profissional e da inserção de tecnologia, dentre as quais a TI.

De forma geral, a abertura econômica da década de noventa trouxe para as empresas do setor produtivo brasileiro a competição em âmbito mundial. Desde então, o avanço da TI, a formação de blocos comerciais, a disseminação de empresas (majoritariamente estrangeiras) ditas globais pelos cinco continentes e os diferentes custos de mão-de-obra e incentivos fiscais entre os países desenvolvidos e em desenvolvimento levaram a uma situação em que qualquer redução significativa nos custos de produção poderia alterar a distribuição das plantas industriais no mundo conforme Muniz (2000).

Nesse contexto é que as empresas brasileiras passaram a responder de maneira mais flexível às mudanças do mercado e a competir com os produtos estrangeiros. Diante desses fatores, 
o investimento em TI deve ser realizado com cautela, pois o simples fato de investir em TI pode não contribuir significativamente para o aumento de produtividade dentro da empresa.

Fatores estruturais da organização foram fundamentais no suporte à TI, e as relações entre investimento em TI e produtividade são complexas. O texto buscará identificar caminhos alternativos para avaliar os benefícios potenciais da TI para as empresas, de acordo com a situação estrutural das empresas e seu alinhamento com a conjuntura global.

\section{Avaliação do suporte oferecido à TI para aumento da produtividade}

a)Verificação dos processos gerenciais

Conceituação bastante satisfatória é dada por Uono (1996), na qual processo é uma ordenação específica de eventos de uma empresa que, consumindo recursos de trabalho no espaço e no tempo e visando um resultado específico, possui um começo e um fim, além de entradas e saídas claramente identificadas e definidas. Para e Hammer e Champy (1994), a gestão por processos apresenta vantagem para a produtividade, pois medir a eficiência por departamento ou por atividades isoladas prejudica a eficiência da organização como um todo.

Tenner (1997) apresenta métodos de desenho de processos que oferecem soluções gerenciais, além de técnicas de intervenção no desempenho das empresas que vão das mais conservadoras, como programas de melhoria contínua, até as mais radicais como a reengenharia, conforme Pereira (1999). Um quadro bastante completo de processos empresariais aplicáveis à maioria das empresas é apresentado por Gonçalves (2000), dos quais alguns são apresentados no quadro 1.

Quadro 1 - Família de processos gerenciais

\begin{tabular}{|l|l|}
\hline \multicolumn{1}{|c|}{ Famillia de processos } & \multicolumn{1}{c|}{ Processos selecionados } \\
\hline Administração Geral & Gerência de contas, comunicações internas, desenv. de lideranças; \\
\hline Manufatura & Gestão da qualidade, gestão de estoques, distribuição; \\
\hline Marketing & Gestão de canal, pesquisa de marketing, introdução de produtos; \\
\hline Educacionais & Desenvolvimento de habilidades, educação do consumidor; \\
\hline Desenvolvimento de tecnologia & Avaliação de tecnologia, seleção de novos produtos; \\
\hline Financeiros & Acompanhamento do desempenho, gestão de crédito de clientes; \\
\hline Organizacionais & Recrutamento e seleção, avaliação de desempenho; \\
\hline Legais & Regulamentação, registro e controle de patentes; \\
\hline Comerciais & Marketing direto, gestão de filiais; \\
\hline De suporte ao cliente & Suporte técnico, garantia e reparo. \\
\hline
\end{tabular}

Fonte: Adaptado de Gonçalves (2000).

A inserção da gestão de processos e a racionalização do nível gerencial que passou a ocorrer na maioria das empresas a partir da década de 90 não dependia especificamente da 
tecnologia, vide Feijó e Carvalho (1994). No entanto, a TI possibilita a melhoria do acesso à informação e da coordenação entre unidades organizacionais em termos de custo e tempo, de forma que as funções dentro da organização, tais como marketing e produção, não deixaram de existir, pois são centros de conhecimento dentro das empresas, mas passaram a alinhar-se no sentido de obter o resultado final para o cliente, convergindo na gestão por processos.

A partir daí, os saltos de produtividade obtidos com a TI e a gestão de processos como ferramenta gerencial podem deixar de ser obtidos apenas com a redução de custos (uma vez que este paradigma gerencial é altamente poupador de mão-de-obra), mas também com técnicas de diferenciação, capacitando as empresas a agregar valor em seus produtos e serviços, tornando-se mais competitivas.

\section{b)Capacitação profissional}

Embora a TI tenha tido como efeito a drástica redução de empregos, permitiu a criação de outros que demandam maior grau de capacitação, vide Laver (1989). Cohen (2003) argumenta que mesmo o papel dos líderes mudou, e para estes a eficiência é criar condições para que outros profissionais sejam eficazes, de forma que sejam capazes de arranjar recursos, informações e conexões certas para realizar o seu trabalho. O desenvolvimento da TI exige que os profissionais sejam mais selecionadores, questionadores e comparadores.

A tecnologia possibilitou Workgroups, shareware e Internet, viabilizou a transformação da concepção de grupos, a estrutura ad hoc e o compartilhamento de recursos de informação, alterando a maneira pela qual pessoas e órgãos se relacionam conforme Caldas (1999). De maneira geral, o trabalho tende a ser menos manual e mais intensivo em informação e em conhecimento, levando à necessidade de novos padrões de trabalho nas empresas.

\section{c)Intensidade de informação e Grau de inovação tecnológica nos processos e produtos}

Baseando-se na matriz de intensidade de informação de Porter e Millar (1985) apresentada na figura 1, as características do processo e do produto em relação à necessidade de informação influenciam o potencial da TI em beneficiar a organização. Uma empresa bem classificada em um dos quadrantes dessa matriz possui maiores indicações de quão necessários são os investimentos a serem realizados em TI. 


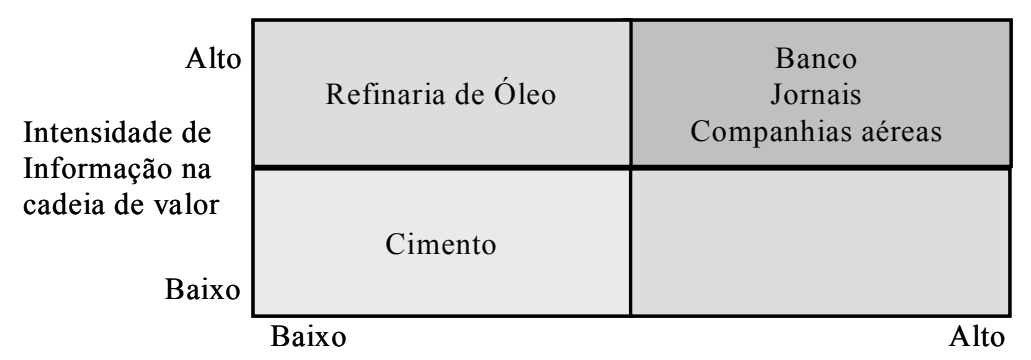

Figura 1 - Intensidade de informação no processo e no produto. Adaptado de Porter e Millar (1985).

\section{d)Especificidades de áreas das empresas}

Para realização de um investimento em TI, é necessário encontrar os fatores críticos para a aplicação da tecnologia para que os investimentos sejam orientados adequadamente.

Uma maneira de orientar os investimentos é como colocado na figura 2, que diferencia os domínios estáveis e os domínios de alta variância da organização, de forma que os sistemas de priorização de eficiência devem ser aplicados nos domínios mais maduros e com pouca

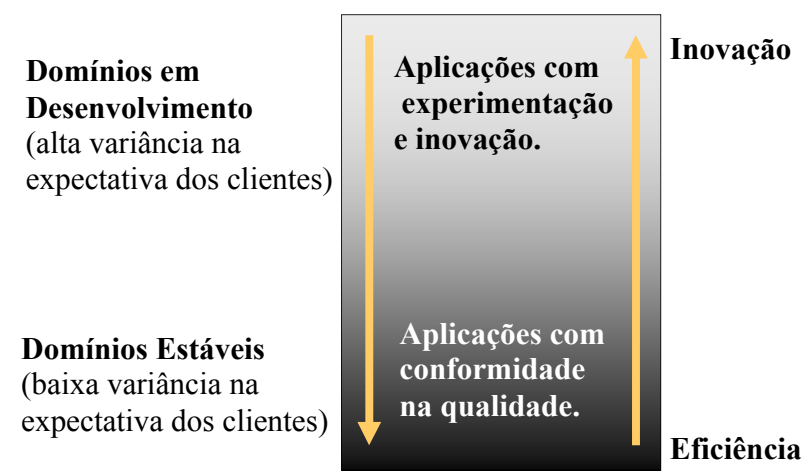

variabilidade.

Figura 2 - Dinâmica do portifólio de aplicações. Adaptado de Prahalad, Krishnan (2002).

Carvalho e Laurindo (2003) descrevem uma outra abordagem igualmente importante, a distinção entre fatores qualificadores (fatores nos quais a empresa deve ter um desempenho mínimo para que continue competindo) e ganhadores de pedidos (fatores que distanciam a empresa da média, melhorando-a em relação aos concorrentes). Dessa maneira a produtividade da organização dependerá da decisão adequada de quais áreas receberão prioritariamente maiores investimentos.

e) Flexibilidade - adequada para o atendimento do mercado

Para Hamel e Välikangas (2003), a flexibilidade, ou a habilidade para reinventar dinamicamente os modelos de negócios e as estratégias conforme as circunstâncias mudam, é uma característica imprescindível para atender a um mercado exigente, global, e altamente mutável. 
Em pesquisa realizada por Weill et al (2002) em empresas norte-americanas de grande sucesso, mostrou-se que embora os componentes de infra-estrutura sejam comumente disponíveis, os processos de gestão necessários para implementá-lo flexivelmente não o são. Cerca de 55\% do orçamento destinado à TI aplica-se tanto na fusão entre tecnologia e processos e quanto no treinamento de pessoas que compõem infra-estrutura. Além disso, tais investimentos envolvem uma escolha entre níveis de retorno presente e futuro, especialmente numa infra-estrutura flexível o suficiente para ser reusada em muitos negócios de maneira a reduzir o time to market.

f)Erros de medição, (Uso de indicadores adequados);

Como visto, produtividade tem dentre outros aspectos tempo, dinheiro, energia. Esta amplitude do conceito de produtividade produziu distorções na medida de produtividade em relação à TI, pois com a entrada da TI alguns dos padrões de medida deveriam também mudar. Willcocks (1996), em seu artigo, argumenta que alguns problemas de medição podem ser evitados através do desenvolvimento de práticas de gestão e avaliação de desempenho em nível gerencial, ou seja, melhoria nas práticas de medição.

Remenyi (2000) apresenta quatro pontos que contribuem para as dificuldades encontradas na medição e no gerenciamento dos benefícios oferecidos pelos investimentos em TI: discernimento entre benefícios tangíveis e intangíveis (há aspectos da TI que são de difícil mensuração, mas que podem impactam no sucesso da organização); evolução do benefício (perspectivas futuras); escopo dos sistemas de informação (integração da TI aos processos organizacionais); identificação das melhorias de performance e benefícios (queda de produtividade no período de implantação do projeto de TI).

\section{Fatores de suporte de produtividade da TI}

Do que foi apresentado acima verifica-se a necessidade de concentrar esforços nos fatores de suporte de produtividade da TI citados abaixo e organizados segundo a figura 3:

a- Adequação dos processos de negócios;

b- Capacitação profisssional adequada e gestão do conhecimento;

c- Verificação da intensidade de informação e do grau de inovação nos produtos e processos;

d- Observação das especificidades de cada área da organização;

e- Flexibilização na estrutura de TI da empresa, de modo a atender rapidamente ao mercado;

f- Indicadores adequados, de forma a evitar erros de medição. 


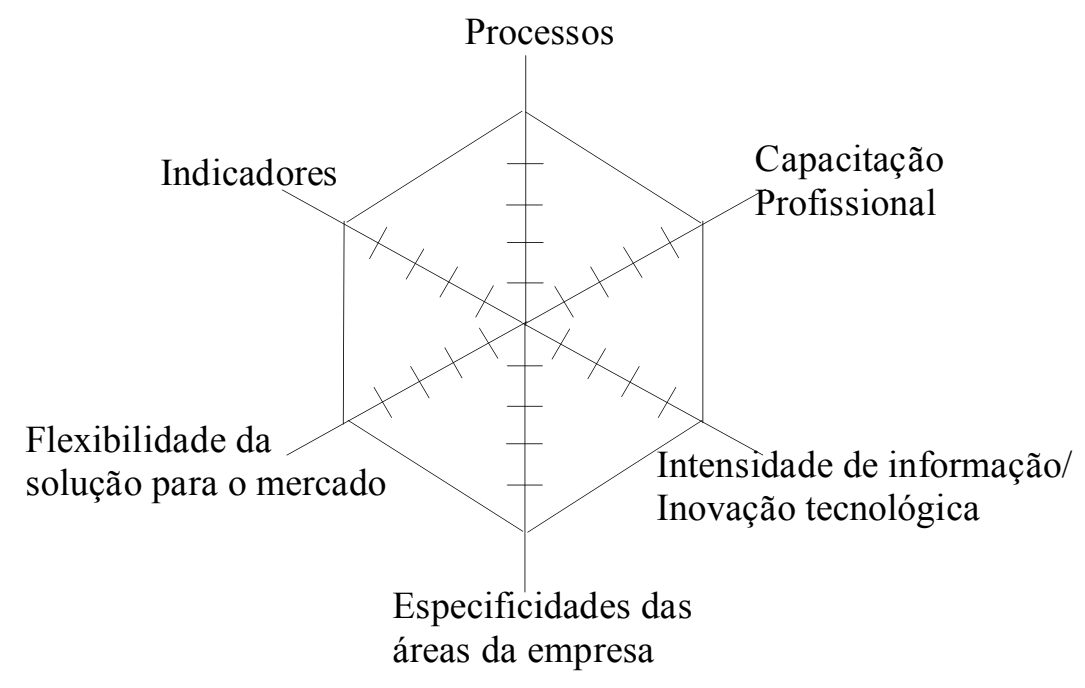

Figura 3 - Fatores de suporte à TI no aumento da produtividade.

\section{Estudo de caso}

A metodologia adotada foi a de estudo de caso conforme Yin (1991), que foi realizado em uma empresa de produção e prestação de serviços na área de cromatografia a gás na cidade de São Paulo, selecionada para devido a estar passando por um processo de revisão em seus processos. As informações foram obtidas através de entrevistas a um dos diretores da empresa.

A empresa originalmente produzia cromatógrafos a gás, que é um tipo particular de analisador de substâncias líquidas volatilizáveis ou substâncias gasosas, largamente utilizado em laboratórios de indústrias químicas, petroquímicas, alimentícias, farmacêuticas e análise ambiental. Seu desenvolvimento envolve profissionais da área química, mecânica, de software, entre outros.

Devido às mudanças que ocorreram no ambiente competitivo brasileiro nos últimos anos, sobretudo a abertura do mercado, a empresa deixou de ter um caráter tipicamente fabril, montando equipamentos com dispositivos construídos pela própria empresa, adquiridos no mercado interno e do mercado externo (componentes e matérias primas), e passou a apresentar também um caráter de prestadora de serviços. Hoje, embora continue a fabricar equipamentos, a receita da empresa provém principalmente de treinamento no uso das máquinas de análise cromatográfica, serviços de 
manutenção, reparação e instalação de equipamentos, fornecimento de peças de reposição e desenvolvimento de métodos de trabalho para laboratórios.

A empresa atua há 40 anos no mercado de cromatografia (praticamente desde o começo da utilização deste tipo de equipamento em laboratórios) e é composta por 15 funcionários dos quais alguns possuem funções bastante flexíveis na empresa, talvez por ser hoje uma empresa de pequeno porte (chegou a ter 140 funcionários antes da abertura do mercado no início dos anos 90). Possui cadastro de atendimento de 140 clientes nos últimos 2 anos, e o preço de um equipamento varia de $\mathrm{R} \$ 20.000,00$ a $\mathrm{R} \$ 300.000,00$.

Foi elaborada uma entrevista com o diretor da empresa, tendo com objetivo investigar os itens apresentados no item 5. Durante a entrevista, falou-se sobre a estrutura existente na empresa que pudesse oferecer benefícios para aplicações de TI conforme a lista proposta. A síntese da análise da entrevista forneceu o seguinte resultado:

a- Adequação dos processos de negócios: Não há processos bem determinados. Como dito anteriormente, muitos funcionários realizam diferentes papéis dentro da empresa, contribuindo também para uma não conformidade dos processos. Como exemplo de alguns processos encontrados, citou-se processo de suporte ao cliente, gerência de contas, avaliação de tecnologia e gestão de qualidade do produto.

b- Capacitação profissional adequada e gestão do conhecimento: Os profissionais da empresa são bem capacitados, pois o trabalho de desenvolvimento de métodos de trabalho para os clientes (um dos serviços prestados) exige grande conhecimento da área técnica tanto do equipamento quanto do negócio do cliente.

c- Intensidade de informação nos produtos e processos: Com o auxílio da matriz de intensidade de informação de PortereMillar (1985), verificou-se que há pouca informação no produto (o produto é simplesmente um equipamento, diferente de um jornal ou banco) mas possui grande intensidade de informação no processo, uma vez que é necessário ter grande conhecimento da necessidade do cliente para a confecção do produto além do conhecimento técnico sobre o produto (os produtos são semi-customizados). Além disso, a empresa tem feito uso intensivo de software em seus equipamentos, permitindo maior grau de inovação em seus produtos e maior flexibilidade para acompanhar as mudanças do mercado.

d- Especificidades das áreas da empresa: A empresa possui duas áreas principais - desenvolvimento de produtos e atendimento ao cliente. Nesse sentido, a área de desenvolvimento de novos produtos possui inerente necessidade de TI (software), característica mais acentuada que na área de atendimento a clientes. 
e- Flexibilidade na estrutura de TI: A empresa percebeu a necessidade da introdução de dispositivos eletrônicos, programados por software. Com isso, percebeu a grande flexibilidade permitida por este recurso no presente e no futuro, pois é possível a reprogramação do sistema para desenvolvimento de novos produtos. Além disso, os produtos são semi-customizados, existindo uma estrutura central e uma variabilidade permitida de acordo com a necessidade do cliente. Esses fatores mostram a preocupação da empresa com a flexibilidade de soluções oferecidas.

f- Indicadores adequados: A empresa utiliza como indicadores o benchmarking e o nível de satisfação dos clientes em relação aos produtos. Seguindo a concepção de Willcocks (1996), estes são bons indicadores de aumento de produtividades.

A figura 4 ilustra a disposição dos fatores de suporte à TI para a promoção da produtividade:

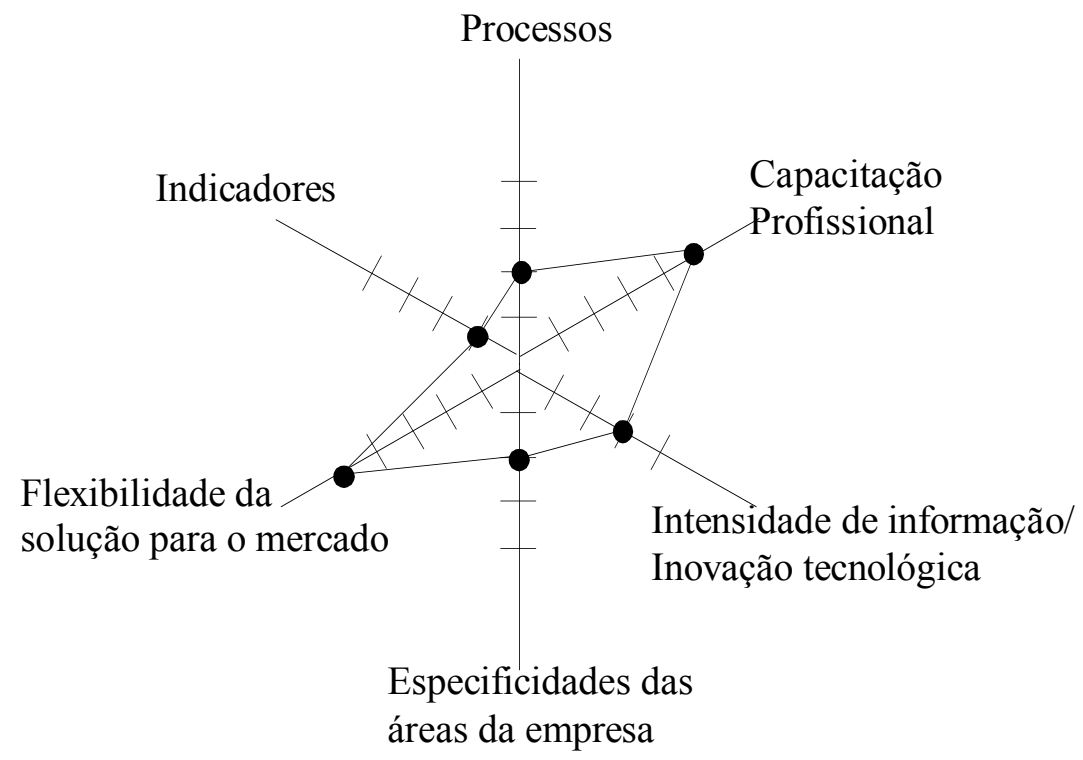

Figura 4 - Alavancas de eficiência para TI

\section{Conclusão}

O texto discorreu sobre alguns aspectos importantes no contexto do suporte necessário à TI dentro das organizações.

Como foi visto, houve um grande salto de produtividade no Brasil na década de 90, explicado pelo fenômeno de reestruturação produtiva ocorrida não só no Brasil mas em nível internacional, altamente poupador de mão de obra e calçada em grande parte pela redução do quadro de funcionários.No entanto, em conjunto com este novo paradigma gerencial houve uma mudança de padrão tecnológico, sendo a TI o aspecto tecnológico mais importante a ser considerado nesse período. 
Dessa maneira, outros aspectos dentro da organização são relevantes para o aumento de produtividade em relação à utilização da TI, os quais foram apresentados no levantamento teórico, chegando-se à lista de verificação apresentada no item 5 e sumarizada na figura 3 . O estudo de caso permitiu compreender melhor como estes fatores devem ser considerados para o uso da TI em prol do aumento de produtividade, trazendo aumento no retorno dos investimentos realizados, além do aumento da confiança na implementação de sistemas empresariais.

\section{Abstract}

The impact of the Information Technology (IT) in the productivity of the management level of the organizations is an important issue that has been extensively studied since the dissemination of IT in the companies in the $70^{\prime}$ s. This effort can be justified by the concern of the companies in relation to the simultaneous pressures suffered, such as the globalization, world-wide changes in the labor market, sophistication of the demand of the consumers, exhaustion of unexplored markets, vide Caldas (1999). However, to measure the productivity of the IT factor is not a simple task, because it depends on subjective variations of each administrator and varies for each company. The solutions use to be generalized for several cases in total different segments, what might generate distortions and unsatisfactory results. The object of study in this article is the set of internal structures of the organization which are capable to extract benefits from IT in favor of the productivity. The research involves a theoretical survey and a case study in a gas chromatography production and service company in São Paulo city. As a result, it was composed a check-list of the necessary elements to stimulate the productivity with the aid of IT.

Key words: Information Technology, Productivity, Business Process

\section{Referências}

CALDAS, M. P. O triste destino da área de O\&M. RAE - Revista de Administração de Empresas, São Paulo, v. 39 n. 2, p. 6-17, abr./jun. 1999.

CARR, N. G. It doesn’t matter. Harvard Business Review, maio 1993.

CARVALHO, M. M.; LAURINDO, F. J. B. Estratégias para competitividade. São Paulo: Futura, 2003.

CHAMPY, J.; HAMMER, M. Reengenharia-revolucionando a empresa. 17. ed. Rio de Janeiro: Campus, 1994.

COHEN, A. Nunca abuse do poder. Exame, p. 62-64, 17 mar. 2004.

FARREL, D. The real new economy. Harvard Business Review, p. 105-112, out. 2003.

FEIJÓ, C.; CARVAlHO, P. G. M. Sete teses equivocadas sobre o aumento da produtividade industrial nos anos recentes. Boletim Conjuntura, Rio de Janeiro, v. 14, n. 2, p. 109-121, 1994.

GONÇALVES, J. E. L. As empresas são grandes coleções de processos. RAE - Revista de Administração de Empresas, São Paulo, v. 40, n. 1, p. 6-19, jan./mar. 2000.

HAMEL, G.; VÄLIKANGAS, L. The quest for resiliense. Harvard Business Review, set. 2003, p. 52-63.

LAURINDO, F. J. B. Tecnologia da Informação: eficácia nas organizações. São Paulo: Futura, 2002.

LAVER, M. Information technology: agent of change. Cambridge: Cambridge University Press, 1989. 
MUNIZ, S. Sambando em gelo fino: investimento industrial, capacitação tecnológica e organização e competitividade brasileira nos anos 90. Tese (Doutorado), Universidade de São Paulo, Departamento de Engenharia de Produção. São Paulo, 2000.

PEREIRA, G. S. R. Uma proposta estratégica para implementação de projetos de reengenharia. Tese (Doutorado), Universidade de São Paulo, Departamento de Engenharia de Produção. São Paulo, 1999.

PORTER, M. E.; MILLAR, V. E. How information gives you competitive advantage. Harvard Business Review, p. 149-160, jul./ago. 1985.

PRAHALAD, C. K.; KRISHNAN, M. S. The Dynamic synchronization of strategy and information technology. MIT Sloam Management Review, Summer 2002.

REMENYI, D. The elusive nature of delivering benefits from IT investment. Disponível em: http://www.ejise.com/volume-3/volume3-issue1/issue1-art1.htm. Acesso em: 23 jan. 2005.

TENNER, A. R.; DE TORO, I. J. Process redesign. Massachussets: Addison Wesley Longman, 1997.

UONO, A. Proposta de uma sistemática para reestruturação de processos. Monografia (Bacharelado), Universidade de São Paulo, Departamentoto Engenharia de Produção. São Paulo, 1996.WEILl, P.; SUBRAMANI, M.; BROADBENT, M. Building IT Infrastructure for Strategic Agility. MIT Sloan Management Review, p. 57-65, out. 2002.

WILLCOCKS, L.; LESTER, S. Beyond IT Productivity Paradox. European Management Journal, v. 14, n. 3, p. 279-290, jun. 1996.

cross ref

YIN, R. Case study research: design and methods. Newbury Park: Sage Pub, 1991. 